\title{
Electronic structure of some -(C10H8S8)2X compounds as studied by infrared spectroscopy
}

\author{
Jacobsen, Claus Schelde; Tanner, D. B.; Williams, Jack M.; Geiser, U.; Wang, H. H.
}

Published in:

Physical Review B

Link to article, DOI:

10.1103/PhysRevB.35.9605

Publication date:

1987

Document Version

Publisher's PDF, also known as Version of record

Link back to DTU Orbit

Citation (APA):

Jacobsen, C. S., Tanner, D. B., Williams, J. M., Geiser, U., \& Wang, H. H. (1987). Electronic structure of some (C10H8S8)2X compounds as studied by infrared spectroscopy. Physical Review B, 35(18), 9605-9613. https://doi.org/10.1103/PhysRevB.35.9605

\section{General rights}

Copyright and moral rights for the publications made accessible in the public portal are retained by the authors and/or other copyright owners and it is a condition of accessing publications that users recognise and abide by the legal requirements associated with these rights.

- Users may download and print one copy of any publication from the public portal for the purpose of private study or research.

- You may not further distribute the material or use it for any profit-making activity or commercial gain

- You may freely distribute the URL identifying the publication in the public portal 


\title{
Electronic structure of some $\beta-\left(\mathrm{C}_{10} \mathrm{H}_{8} \mathrm{~S}_{8}\right)_{2} X$ compounds as studied by infrared spectroscopy
}

\author{
C. S. Jacobsen and D. B. Tanner* \\ Physics Laboratory III, Technical University of Denmark, DK-2800 Lyngby, Denmark \\ Jack M. Williams, U. Geiser, and H. H. Wang \\ Chemistry Division and Materials Science Division, Argonne National Laboratory, Argonne, Illinois 60439-4843
}

(Received 17 December 1986)

\begin{abstract}
Polarized reflectance measurements have been made on two isostructural conducting compounds of bis(ethylenedithio)tetrathiafulvalene [BEDT-TTF or ET, $\left.\left(\mathrm{C}_{10} \mathrm{H}_{8} \mathrm{~S}_{8}\right)\right]: \quad \beta$-(ET) ${ }_{2} \mathrm{AuI} \mathrm{I}_{2}$ and $\beta$ (ET) ${ }_{2} \mathrm{I}_{2} \mathrm{Br}$. The former is superconducting at ambient pressure with $T_{c}=5 \mathrm{~K}$, whereas the latter retains normal-metal conductivity to low temperatures. The reflectance measurements, made at temperatures of approximately 30 and $300 \mathrm{~K}$, spanned $80 \mathrm{~cm}^{-1}(0.01 \mathrm{eV})$ through $33000 \mathrm{~cm}^{-1}(\sim 4$ $\mathrm{eV}$ ); they were made for polarization along the ET molecular stacking axis and transverse to it in the sheets or layers of ET molecules. Band-structure parameters determined from the plasmon frequencies imply that the anisotropy is rather low for organic conductors, with $t_{\|} \approx 0.22 \mathrm{eV}$ and $t_{1} \approx 0.09$ $\mathrm{eV}$ for $\beta-(\mathrm{ET})_{2} \mathrm{AuI}_{2} ; t_{\|} \approx 0.18 \mathrm{eV}$ and $t_{1} \approx 0.07 \mathrm{eV}$ for $\beta-(\mathrm{ET})_{2} \mathrm{I}_{2} \mathrm{Br}$. At $300 \mathrm{~K}$, the spectra differ substantially from the expectations of simple one-electron models, suggesting that both electronphonon and electron-electron interactions play important roles in the electronic structure of these materials. At low temperatures, the materials display basically metallic characteristics, yet deviate significantly from simple Drude-model behavior. At $30 \mathrm{~K}$, no effect attributable to superconducting fluctuations could be observed.
\end{abstract}

\section{INTRODUCTION}

In the study of superconductivity in organic solids, a particular group of materials based on the molecule bis(ethylenedithio)tetrathiafulvalene $\quad\left[\mathrm{C}_{10} \mathrm{~S}_{8} \mathrm{H}_{8} \quad\right.$ (BEDTTTF or in short, ET)] has attracted considerable interest in the last few years. The materials in focus are of the sort $(\mathrm{ET})_{n} X_{m}$, where $X$ can be one of several inorganic monovalent anions and $n$ and $m$ are in the range $1-3$. In $1982(\mathrm{ET})_{2} \mathrm{ClO}_{4}-\left(\mathrm{C}_{2} \mathrm{H}_{3} \mathrm{Cl}_{3}\right)_{0.5}$ (a compound which also contains trichloroethane solvent molecules) was reported ${ }^{1}$ metallic to $1.4 \mathrm{~K}$. Superconductivity was first detected ${ }^{2}$ in a particular phase of $(\mathrm{ET})_{2} \mathrm{ReO}_{4}$, at pressures above 4.5 kbar and temperatures below $2 \mathrm{~K}$. The first ambient pressure superconductor in the ET family was discovered in 1983, when $(E T)_{2} I_{3}$ in its triclinic $\beta$ phase was reported ${ }^{3}$ to have a transition temperature $T_{c} \sim 1.4 \mathrm{~K}$. Since then it has been found ${ }^{4}$ that $T_{c}$ is extremely pressure sensitive, increasing to $\sim 8 \mathrm{~K}$ at $>0.5 \mathrm{kbar}$. It has been shown $\mathrm{n}^{5-7}$ that $T_{c}$ remains high $(8 \mathrm{~K})$ if the pressure is released at low tempertures and the samples are not warmed above $125 \mathrm{~K}$. Other ambient-pressure superconductors based on ET include $\left.{ }^{8-10} \gamma-(\mathrm{ET})_{2}(\mathrm{I})_{3}\right)_{2.5}\left(T_{c} \cong 2.5 \mathrm{~K}\right), \beta-(\mathrm{ET})_{2} \mathrm{IBr}_{2}$ $\left(T_{c} \cong 2.8 \mathrm{~K}\right)$, and $\beta$-(ET) ${ }_{2} \mathrm{AuI}_{2}\left(T_{c} \cong 5 \mathrm{~K}\right)$. All these findings were rather surprising because in the $\beta$-(ET) ${ }_{2} X$ family the room-temperature conductivity is rather low, ${ }^{3}$ about $30 \Omega^{-1} \mathrm{~cm}^{-1}$, corresponding to conduction-electron mean free paths much shorter than a lattice constant. Ordinarily, organic conductors with such low room-temperature conductivities become insulating at low temperatures.

As in other organic conductors, the ET molecules are organized in stacks, with considerable intrastack $\pi$-orbital overlap. The anions are closed shell and do not contribute to the low-frequency conductivity. An additional feature of the ET compounds follows from the large number of sulfur atoms on the ET molecule and the many short $\left(d_{\mathrm{S}-\mathrm{S}}<3.60 \AA\right.$, the van der Waals radius sum of $\left.\mathrm{S}\right)$ interstack contacts. ${ }^{1,2,11,12}$ In the $\beta$-phase compounds the interstack contacts are short and the conductivity ${ }^{3}$ and thermoelectric power ${ }^{13}$ vary by no more than a factor of 2 in the $a-b$ plane, the plane which contains the sheets of interacting ET chains. Other important features ${ }^{11}$ of these 2:1 compounds are (1) an organization of the stack in interacting dimers, which may just be a consequence of the stoichiometry, and (2) a tilt of the normal to the ET molecules away from the stacking axis $\left[\sim 28^{\circ}\right.$ in $\left.\beta-(\mathrm{ET})_{2} \mathrm{AuI}_{2}\right]$.

The electronic band structure may either be thought of as quarter-filled holelike referring to one ET molecule as a unit, but then with a considerable dimerization gap in the middle, or more correctly as a half-filled band based on the dimer as a unit and with a very close lying, filled lower band. Available band-structure calculations ${ }^{14,15}$ suggest a closed, cylindrical Fermi surface.

Previous infrared and optical studies on $\beta$-(ET) $)_{2} X$ compounds have mostly centered on the room-temperature properties of the $\mathrm{I}_{3}{ }^{-}$compound, ${ }^{16-20}$ but the discovery ${ }^{21}$ of qualitative changes with temperature has spurred further investigations. ${ }^{22,23}$ All studies report pronounced quasimetallic behavior in the stacking direction at $300 \mathrm{~K}$ (a well-defined plasma edge), but overdamped behavior perpendicular to the stacks (slow rise in reflectance towards low frequencies). At low temperatures plasma edges are found in both polarizations, ${ }^{22-24}$ but the spectra do not follow simple Drude behavior.

The aim of the present paper is to present optical spectra extending to far infrared frequencies, for two novel members of the isostructural $\beta$-(ET) ${ }_{2} X$ family, namely ${ }^{10}$ 
$\beta$-(ET) $)_{2} \mathrm{AuI}_{2}$ and ${ }^{25} \quad \beta$-(ET) ${ }_{2} \mathrm{I}_{2} \mathrm{Br}$. The $\mathrm{AuI}_{2}{ }^{-}$, or diiodoaureate, anion is a linear, symmetric, pseudohalide,

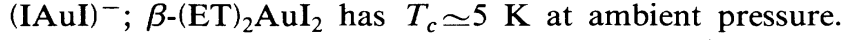
In contrast, $\beta$ - $(\mathrm{ET})_{2} \mathrm{I}_{2} \mathrm{Br}$ has a nonsuperconducting (measured to $0.45 \mathrm{~K}$ by rf penetration ${ }^{25}$ ), metallic ground state. The absence of superconductivity in the latter material is believed to be due to disorder in the orientation of the asymmetric $\mathrm{I}_{2} \mathrm{Br}^{-}$ions, ${ }^{25}$ i.e., $(\mathrm{IIBr})^{-}$or $(\mathrm{BrII})^{-}$. We shall further compare the results with earlier data ${ }^{21}$ on $\beta$ $(\mathrm{ET})_{2} \mathrm{I}_{3} \quad\left(T_{c} \cong 1.4 \mathrm{~K}\right.$, ambient pressure). A preliminary presentation of our measurements has appeared. ${ }^{24}$

\section{EXPERIMENT}

All measurements were done on individual single crystals of the distorted hexagon morphology, electrocrystallized from trichloroethane or tetrahydrofuran. ${ }^{12}$ The samples had high-quality optical (001) faces of typical areas $1-2 \mathrm{~mm}^{2}$.

These highly opaque samples are conveniently studied by means of near-normal-incidence, polarized reflectance measurements. The optical axes in the infrared range were determined as those directions yielding the maximum anisotropy when the polarizer was rotated. As previously found, ${ }^{16,21-23}$ these axes correspond to the stacking axis $\mathbf{a}+\mathbf{b}(||)$, and a direction in the $(a, b)$ plane perpendicular to the stacks $(\perp)$. All subsequent measurements were carried out along these directions, although in the triclinic crystals in question the principal axes may well rotate at high frequencies, where intramolecular excitations in ET and/or $\mathrm{ET}^{+}$and counterions ${ }^{20}$ dominate the spectra. However, the intraband absorption which involves the charge carriers is at much lower frequencies than these high-frequency absorptions.

Data were accumulated in the $60-35000-\mathrm{cm}^{-1}$ frequency range using three different instruments. The far infrared $60-700 \mathrm{~cm}^{-1}$, was covered with a Bruker IFS113V Fourier-transform spectrometer equipped with an A510 reflectance attachment, a wire grid polarizer on polyethylene, and $\mathrm{He}$-cooled $\mathrm{Ge}$ bolometer. A PerkinElmer model 98 grating monochromator was used in the $400-5000 \mathrm{~cm}^{-1}$ mid infrared range with conventional mirror optics, a grid polarizer on $\mathrm{AgBr}$, and a Golay cell detector. Finally, the high-frequency range was covered with a low-resolution Perkin-Elmer 98 fused quartz prism monochromator with Glan-Thompson prism polarizers, and $\mathrm{PbS}$ cell/photomultiplier as detectors. Gold and aluminum mirrors were used as reference in the infrared and visible, respectively.

Low-temperature spectra were obtained by thermally anchoring the sample to a temperature-controlled cold finger. Radiation shields with suitable holes reduced the amount of $300-K$ radiation reaching the samples, but in the present measurements the lowest attainable sample temperatures were ${ }^{26} \sim 30 \mathrm{~K}$.

\section{RESULTS}

\section{A. Reflectance data}

In Fig. 1 we show the broadband room-temperature reflectance of $\beta$-(ET) $)_{2} \mathrm{I}_{2} \mathrm{Br}$. For $\|$ as well as $\perp$ polarizations

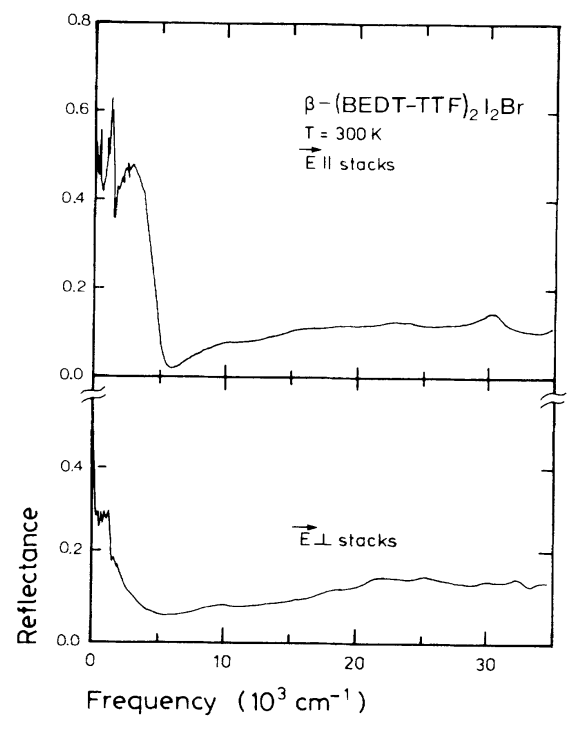

FIG. 1. Polarized reflectance of $\beta$ - $(E T)_{2} I_{2} \mathrm{Br}$ from the far infrared to the near ultraviolet $(T=300 \mathrm{~K})$.

the reflectance is fairly low $(<15 \%)$ above $\sim 6000 \mathrm{~cm}^{-1}$ with a number of weak dispersions at $10 \times 10^{3}, 16 \times 10^{3}$ $23 \times 10^{3}$, and $30 \times 10^{3} \mathrm{~cm}^{-1}$ for $\|$ and $10 \times 10^{3}, 18 \times 10^{3}$, $22 \times 10^{3}, 25 \times 10^{3}$, and $32 \times 10^{3} \mathrm{~cm}^{-1}$ for $\perp$. Approaching low frequencies, a well-developed plasma edge is present in the || polarization, but it is noted that the vibronic structure below $2000 \mathrm{~cm}^{-1}$ is very pronounced. For the $\perp$ polarization the rise is more gradual, corresponding to overdamped plasmon behavior where the relaxation rate is comparable to or exceeds the plasmon frequency. The overall features are typical for all the compounds.

In the following we describe the infrared part of the reflectance spectra as a function of material and temperature. Figures 2 (a) and 2(b) present the stacking axis reflectance to $8000 \mathrm{~cm}^{-1}$ for $\beta$-(ET) ${ }_{2} \mathrm{AuI}_{2}$ and $-\mathrm{I}_{2} \mathrm{Br}$ at $T=300$ and $30 \mathrm{~K}$. Common features include the plasma edge around $5000 \mathrm{~cm}^{-1}$, which sharpens and is slightly blue-shifted on cooling, and pronounced non-Drude structure in the range of molecular vibrations $\left(<2000 \mathrm{~cm}^{-1}\right)$. Differences between the two materials are (1) a higher position of the plasma edge in the $\mathrm{AuI}_{2}{ }^{-}$material, and (2) in the same compound a higher reflectance level below 2000 $\mathrm{cm}^{-1}$ at $30 \mathrm{~K}$. Also, the detailed shape in the vibrational lines differs, although, for example, the rather strong, split bands at $\sim 400 \mathrm{~cm}^{-1}$ and $\sim 1300 \mathrm{~cm}^{-1}$ are present in both $300-\mathrm{K}$ spectra.

In Figs. 3(a) and 3(b) we show the results for $\mathbf{E} \perp$ stacks. The overdamped response at $300 \mathrm{~K}$ changes at $30 \mathrm{~K}$ to a reasonably well-defined plasma minimum in both materials, at $4000 \mathrm{~cm}^{-1}$ in the $\mathrm{AuI}_{2}^{-}$and at $3500 \mathrm{~cm}^{-1}$ in the $\mathrm{I}_{2} \mathrm{Br}^{-}$compounds, respectively. The former shows pronounced non-Drude behavior at $30 \mathrm{~K}$ below $1000 \mathrm{~cm}^{-1}$, whereas the latter displays a gradual rise in reflectance with decreasing frequency, more in accordance with expectations for a metal. The chain-axis vibrational line near $400 \mathrm{~cm}^{-1}$ is absent in all transverse spectra, whereas 

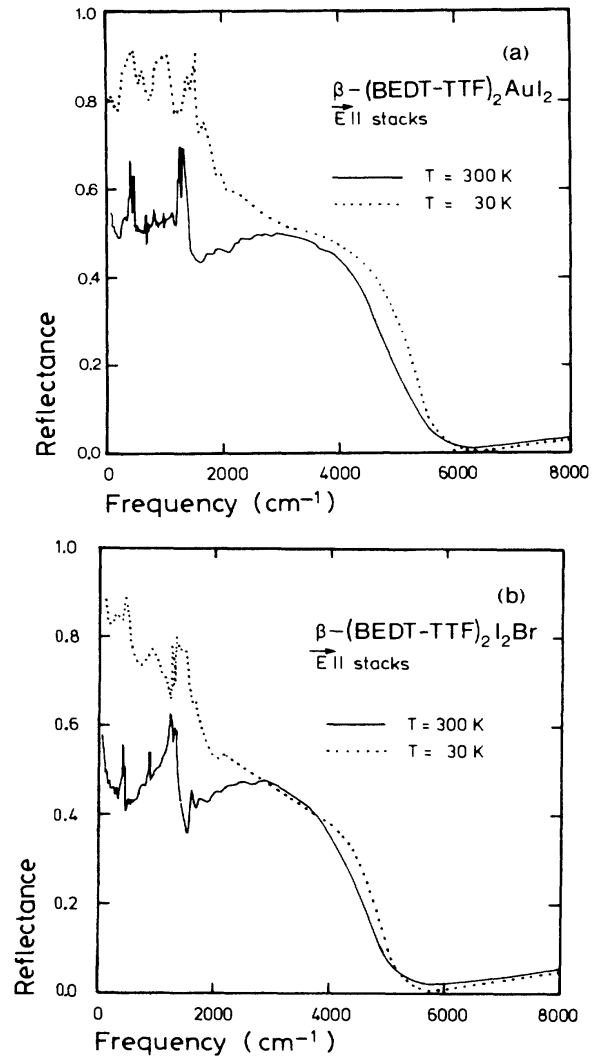

FIG. 2. Infrared stacking axis reflectance of (a) $\beta$-(ET) ${ }_{2} \mathrm{AuI}_{2}$ and (b) $\beta$-(ET) ${ }_{2} \mathrm{I}_{2} \mathrm{Br}$, shown at two temperatures, $T=30$ and 300 $\mathrm{K}$, respectively.

the $1300 \mathrm{~cm}^{-1}$ structure is present in the $300-\mathrm{K} \mathrm{I}_{2} \mathrm{Br}$ data although weaker than along the stacks.

Figures 4 and 5 show details of the far-infrared reflectance as a function of temperature. In both polarizations it is evident that the $\mathrm{AuI}_{2}{ }^{-}$data show distinct deviations from simple Drude behavior in the frequency range below $400 \mathrm{~cm}^{-1}$. For $\mathbf{E}||$ stacks it is noteworthy how the strong doublet at $430-460 \mathrm{~cm}^{-1}$ is barely visible at low temperatures. This apparent change need not signal a change in oscillator strength, but may well be due to the change in background dielectric function (see below). For $\mathbf{E} \perp$ stacks the complete absence of the doublet is again noted. Apart from the non-Drude features discussed already, it is also clear that the $400-500 \mathrm{~cm}^{-1}$ level rises faster with decreasing temperature in the $\mathrm{AuI}_{2}{ }^{-}$compound than in the $\mathrm{I}_{2} \mathrm{Br}^{-}$compound.

\section{B. Kramers-Kronig analysis: $\sigma$ and $\epsilon$}

The polarized reflectance spectra presented above have been analyzed by means of Kramers-Kronig transformation with the phase shift on reflection calculated according to $^{27}$

$$
\theta(\omega)=\frac{\omega}{\pi} P \int_{0}^{\infty} \frac{\ln R\left(\omega^{\prime}\right)}{\omega^{2}-\left(\omega^{\prime}\right)^{2}} d \omega^{\prime}
$$
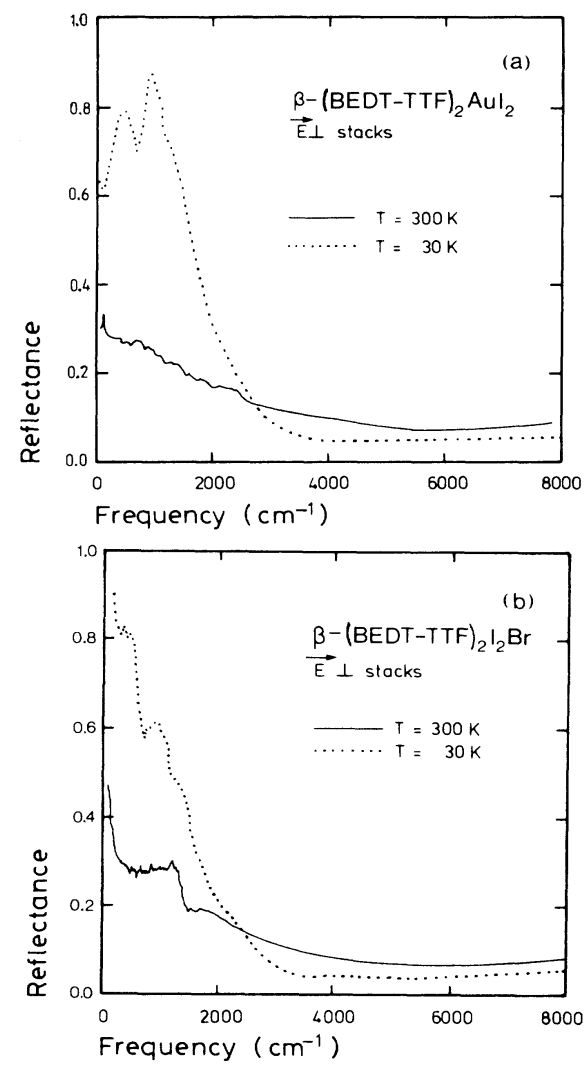

FIG. 3. Infrared reflectance for $\mathbf{E} \perp$ stacks of (a) $\beta$-(ET) ${ }_{2} \mathrm{AuI}_{2}$ and (b) $\beta$-(ET) ${ }_{2} \mathrm{I}_{2} \mathrm{Br}$, shown at two temperatures, $T=30$ and 300 $\mathrm{K}$, respectively.

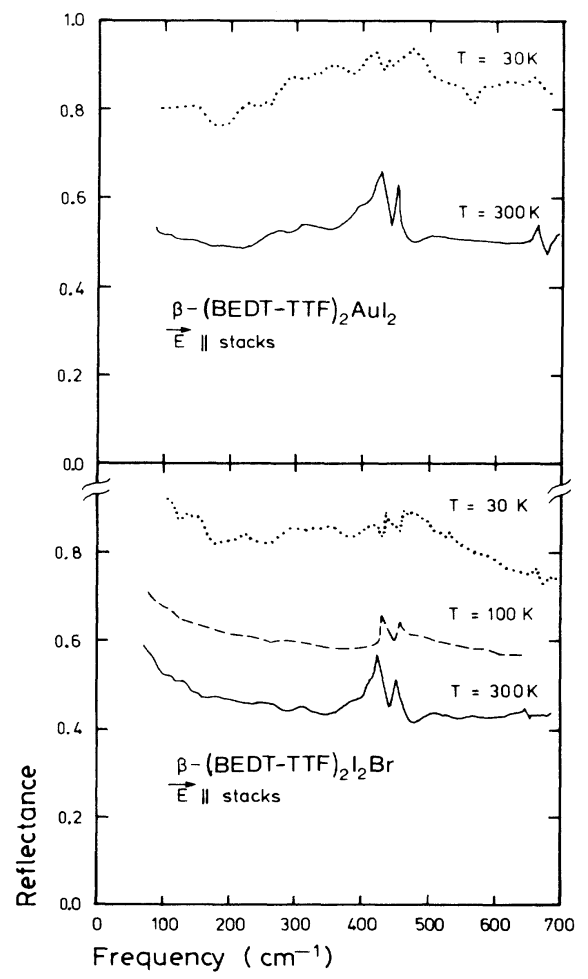

FIG. 4. Far-infrared stacking axis reflectance of $\beta$-(ET) $)_{2} \mathrm{AuI}_{2}$ and $\beta-(\mathrm{ET})_{2} \mathrm{I}_{2} \mathrm{Br}$, for temperatures from 30 to $300 \mathrm{~K}$. 


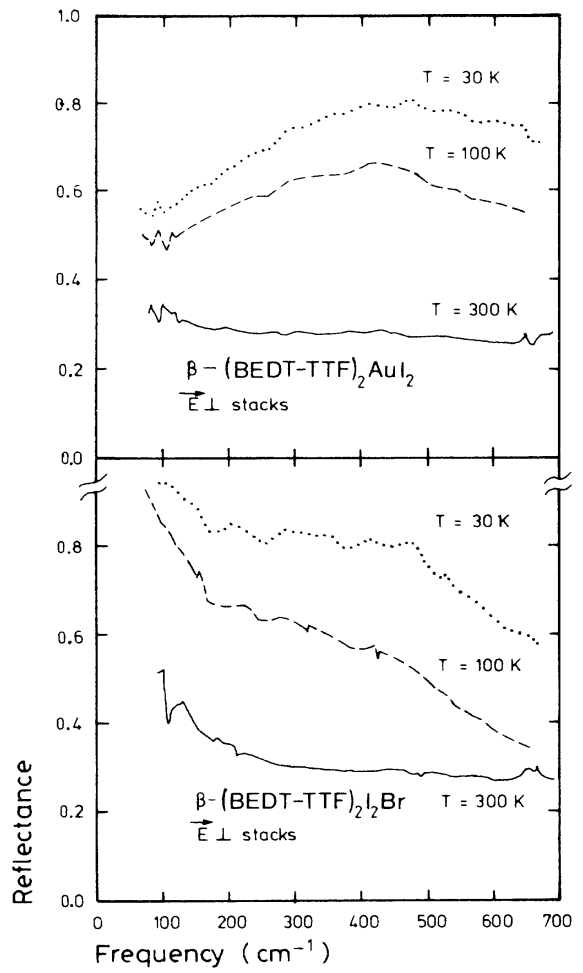

FIG. 5. Far-infrared reflectance for $\mathbf{E} \perp$ stacks of $\beta$-(ET) ${ }_{2} \mathrm{AuI}_{2}$ and $\beta$-(ET) ${ }_{2} \mathrm{I}_{2} \mathrm{Br}$, for temperatures from 30 to 300 K.

The response functions $\epsilon(\omega)$ and $\sigma(\omega)$ are then given by

$$
\epsilon(\omega)+i \frac{\sigma(\omega)}{\omega \epsilon_{0}}=\frac{1+\sqrt{R(\omega)} e^{i \theta(\omega)}}{1-\sqrt{R(\omega)} e^{i \theta(\omega)}} .
$$

In Eq. (1) it is in principle necessary to know $R(\omega)$ at all frequencies. The present study covers a frequency range of approximately 2.5 orders of magnitude around the range of primary interest. With Hagen-Rubens [Drudelike: $R(\omega)=1-A \omega^{1 / 2}$ ] extrapolations at low frequency, and representative extrapolations at high frequencies $\left[R(\omega) \propto\left(\omega_{0} / \omega\right)^{d}, \quad d=0-1\right.$, to $10^{5} \mathrm{~cm}^{-1}$ and $d=4$ beyond], the results in the infrared are expected to be rather reliable. ${ }^{27}$ The position of absorption bands should also be well-reproduced beyond the infrared, but the intensity distribution (oscillator strength) in that region may be influenced by the details of the extrapolation scheme adopted.

As an example of the broad frequency behavior we present again the $300-\mathrm{K}$ results for $\beta$-(ET) ${ }_{2} \mathrm{I}_{2} \mathrm{Br}$, now as conductivity from $80-34000 \mathrm{~cm}^{-1}$, Fig. 6. The band below $8000 \mathrm{~cm}^{-1}$ in both polarizations is the basic absorption related to the dc conductivity. This band is discussed in more detail below. The absorption features from $16000 \mathrm{~cm}^{-1}$ and up may with a fair degree of certainty be assigned to internal, excitonlike transitions in either ${ }^{20} \mathrm{ET}^{+}$or $\mathrm{I}_{2} \mathrm{Br}^{-}$. The lowest molecular $\mathrm{ET}^{+}$excitations are expected to be polarized in the plane of the molecule. We note that the long axis of the molecule has no

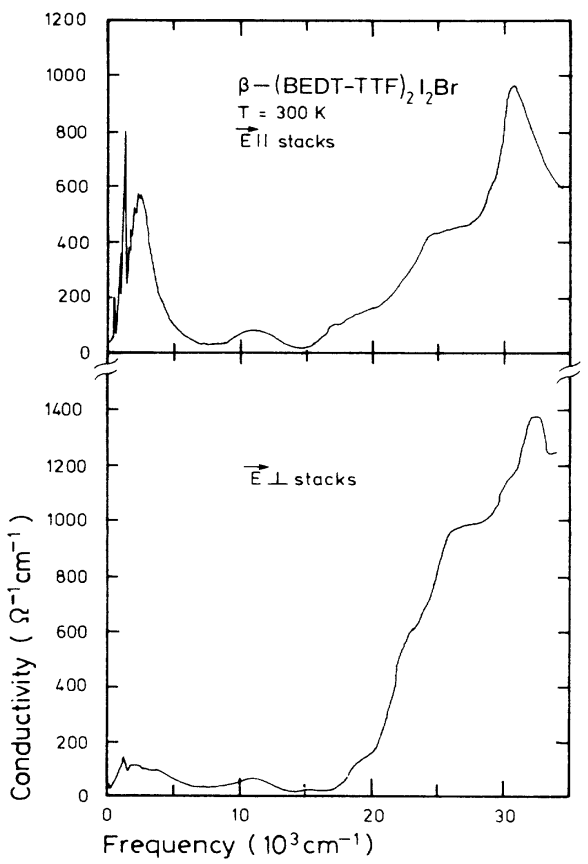

FIG. 6. Frequency-dependent conductivity of $\beta$-(ET) ${ }_{2} \mathrm{I}_{2} \mathrm{Br}$ from the far infrared to the near ultraviolet. Data are shown for $T=300 \mathrm{~K}$ and for $\mathbf{E} \|$ stacks and $\mathbf{E} \perp$ stacks.

component along the $\perp$ polarization. Thus the bands observed in that polarization (at $\sim 19 \times 10^{3}, 23 \times 10^{3}$, $26 \times 10^{3}$ and $33 \times 10^{3} \mathrm{~cm}^{-1}$ ) are presumably due to absorption in $\mathrm{I}_{2} \mathrm{Br}^{-}$or to higher lying transitions in $\mathrm{ET}^{+}$ with polarization perpendicular to the long axis. The bands polarized parallel to the chains (at $\sim 16 \times 10^{3}$, $24 \times 10^{3}$, and $31 \times 10^{3} \mathrm{~cm}^{-1}$ ) may similarly be assigned to long-axis transitions or to $\mathrm{I}_{2} \mathrm{Br}^{-}$excitations.

The origin of the $11000-\mathrm{cm}^{-1}$ band observed in both polarizations is less clear. One suggestion is that such low-lying bands in general ${ }^{28}$ can be interpreted as originating from charge-transfer processes creating doubly occupied states (thus basically measuring the on-site Coulomb interaction $U$ ). However, for the low charge density in question, the intensity of this band should be vanishing, ${ }^{29}$ and it is indeed not observed in systems such as (TMTSF) ${ }_{2} X$ and (TMTTF) ${ }_{2} X$, which have similar carrier densities. The main structural difference is that the organic molecules are nearly perpendicular to the stacks in the latter materials in contrast to the situation here. This difference hints that the $11000-\mathrm{cm}^{-1}$ band arises from mixing between the charge-transfer process and low-lying intramolecular excitations. Such an explanation has previously been proposed for similar absorptions in TCNQ compounds. ${ }^{30}$

In the following we shall concentrate on the infrared part of the spectrum. The frequency dependent conductivity is shown in Figs. 7(a) and 7(b) and 8(a) and 8(b) for the two materials, two temperatures, and two polarizations. In both materials the stacking axis spectrum has about three times as much oscillator strength as the trans- 
verse one. Another common feature is evident in all these results: At room temperature $\sigma(\omega)$ shows a broad peak around $2200 \mathrm{~cm}^{-1}$ with vibrational structure on the lowfrequency side. At low temperature the oscillator strength moves down in frequency so the spectra become more Drude-like. Although there is still sharp fine structure, $\sigma(\omega)$ peaks well below $500 \mathrm{~cm}^{-1}$. The room-temperature spectra for the two materials are rather similar, except for the absence of significant fine structure in the 1500-2000 $\mathrm{cm}^{-1}$ range for $\beta$-(ET) ${ }_{2} \mathrm{AuI}_{2}, \mathrm{E} \perp$ stacks. At low temperature $\sigma(\omega)$ reaches higher conductivity levels in $\beta$ (ET) ${ }_{2} \mathrm{AuI}_{2}$, but this material also shows the most marked deviations from Drude behavior at very low frequencies (far infrared).

The characteristic change with temperature of the spec-
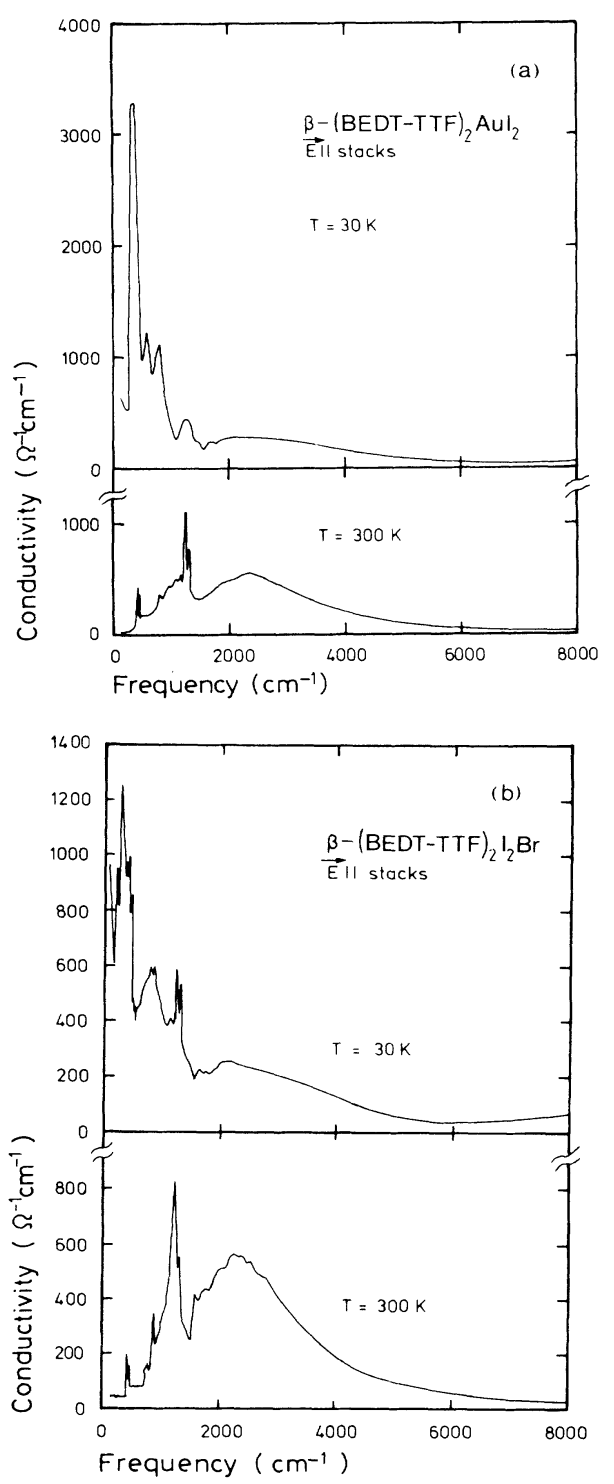

FIG. 7. Infrared stacking axis conductivity of (a) $\beta$-(ET) ${ }_{2} \mathrm{AuI}_{2}$ and (b) $\beta$-(ET) ${ }_{2} \mathrm{I}_{2} \mathrm{Br}$, shown at two temperatures, $T=30$ and $300 \mathrm{~K}$, respectively.
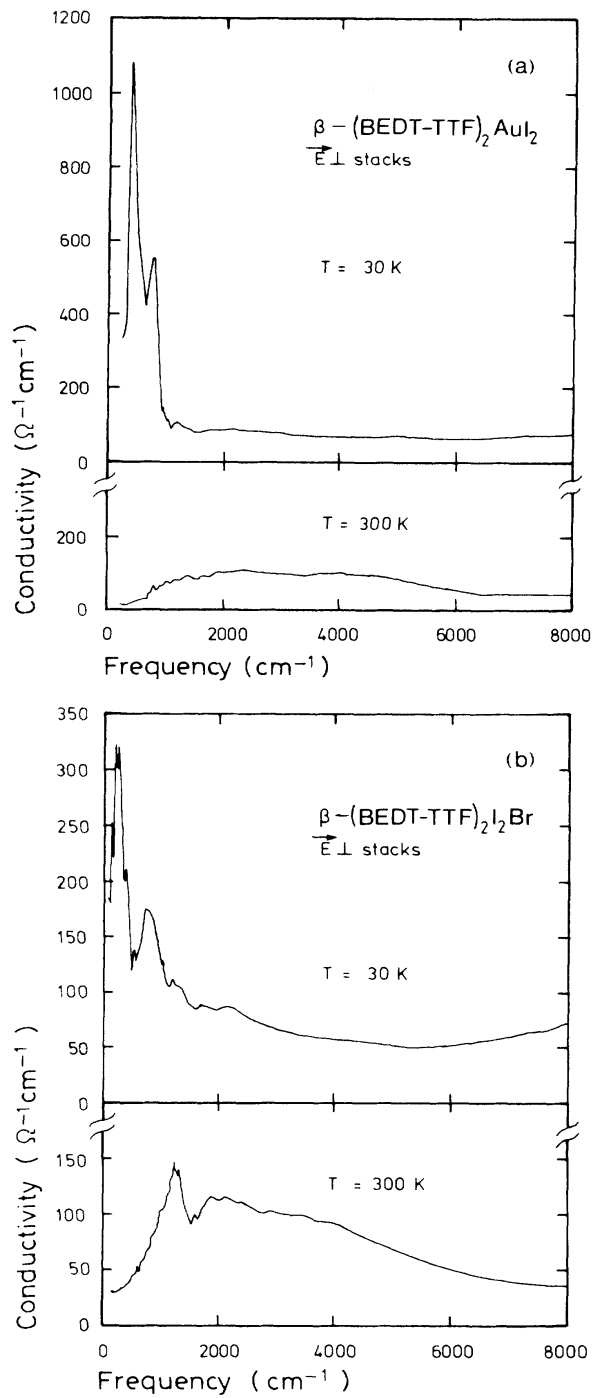

FIG. 8. Infrared conductivity for $\mathbf{E} \perp$ stacks of (a) $\beta$-(ET) ${ }_{2} \mathrm{AuI}_{2}$ and (b) $\beta$-(ET) $)_{2} \mathrm{I}_{2} \mathrm{Br}$, shown at two temperatures, $T=30$ and $300 \mathrm{~K}$, respectively.

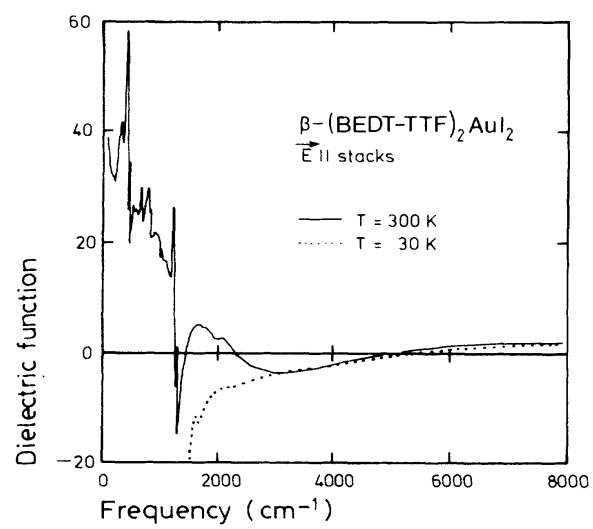

FIG. 9. Stacking axis dielectric function for $\beta$-(ET) $)_{2} \mathrm{AuI}_{2}$ in the infrared, at two temperatures, $T=30$ and $300 \mathrm{~K}$, respectively. 
tra, previously noted in $^{21} \beta-(\mathrm{ET})_{2} \mathrm{I}_{3}$, is also reflected in $\epsilon(\omega)$, exemplified by the $\mathbf{E}||$ stacks spectra in $\beta$ - $(\mathbf{E T})_{2} \mathrm{AuI}_{2}$ in Fig. 9. The 300-K spectrum is semiconductorlike with a number of zero crossings and positive values at low frequencies. In contrast, the $30-\mathrm{K}$ spectrum only displays a single zero crossing (in the plasma edge region) and stays negative at lower frequencies. This negative $\epsilon(\omega)$ is the strongest evidence that at low temperatures these compounds have a basically metallic character. The zero crossing, identifying the plasmon frequency, only increases $\sim 5 \%$ on cooling in spite of the rather drastic changes at low frequencies.

\section{DISCUSSION}

\section{A. Band structure and infrared properties}

In contrast to most other highly conducting organic crystals, the molecular chains in $\beta$ - $(\mathrm{ET})_{2} \mathrm{X}$ materials show a fair degree of dimerization. Thus, to think of them as quarter-filled band systems with a small dimerization gap at midband may not be appropriate. ${ }^{14,15}$ Indeed, in Ref. 17 the infrared properties of $\beta$ - $(\mathrm{ET})_{2} \mathrm{I}_{3}$ were analyzed in terms of a half-filled conduction band based on the ET dimer as the fundamental unit. Both intraband and interband (across the dimerization gap) contributions are found in this model, with the broad peak at $300 \mathrm{~K}$ in $\sigma(\omega)$ attributed to the interband portion. This peak occurs at an energy which can be explained in terms of a band-structure model, but the model also predicts the intraband (Drudelike) contribution to be considerably bigger than observed, when the data are extended to the far infrared (Ref. 21 and this work). Thus the broad structure at $300 \mathrm{~K}$ in the $2000-3000 \mathrm{~cm}^{-1}$ range appears to possess a larger fraction of the oscillator strength than expected from oneelectron theory. At low temperature we find (Figs. 7 and 8) that at least half of the oscillator strength in the broad band shifts to lower frequencies. The remaining part could very well be due to the interband transitions, 22 but the shifting part must be given a different interpretation.

Here we will take the view that organic conductors such as the ET compounds are fairly strongly interacting systems with respect to the short-range electron-electron and electron-phonon interactions. Evidence for this interpretation comes from the quite low $300-\mathrm{K}$ dc conductivity (mean free path for carriers much shorter than a lattice constant) and from the non-Drude frequency dependent conductivity, which resembles that of Peierls insulators, ${ }^{31}$ Hubbard-type Wigner crystals, ${ }^{32}$ or small polaron systems. ${ }^{33}$ Organic conductors have a variety of external as well as internal (i.e., molecular vibration type of) phonons coupled to the electrons, which in combination with an appreciable short range Coulomb repulsion could lead to an efficient localization of the carriers on the dimers in the high-temperature regime. The true difficulty here is in understanding the transition to excellent metallic behavior with delocalized carriers at low temperature and the approximate $T^{2}$ dependence in the low frequency conductivity over the entire temperature range. ${ }^{34}$
In analyzing the infrared spectra, we will first neglect the anomalous behavior and assume that the response near the plasmon frequency is insensitive to the non-Drude behavior so often observed at low frequencies [cf. the $\epsilon(\omega)$ temperature dependence discussed above]. This approach usually leads to reasonable band-structure parameters ${ }^{35}$ within a rather crude band-structure model.

Thus, we neglect all details and simply introduce average transfer integrals, $t_{\|}$and $t_{\perp}$ in an orthorhombic model:

$$
E\left(k_{\|}, k_{\perp}\right)=-2 t_{\|} \cos \left(k_{\|} d_{\|}\right)-2 t_{\perp} \cos \left(k_{\perp} d_{\perp}\right) .
$$

Here, $d_{\|}$is the mean molecular repeat distance along the stacks and $d_{\perp}$ the stack repeat distance perpendicular to the stacks in the $(a, b)$ plane. Obviously $t_{\|}$is an average value, and $t_{\perp}$ is a weighted average of several interchain transfer integrals appearing in a more detailed triclinic band model. However, $t_{\|}$and $t_{\perp}$ should still fairly represent the overall degree of anisotropy.

We proceed by fitting the Drude model for reflectance:

$$
R(\omega)=\left|\frac{\sqrt{\tilde{\epsilon}(\omega)}-1}{\sqrt{\tilde{\epsilon}(\omega)}+1}\right|^{2},
$$

with

$$
\widetilde{\boldsymbol{\epsilon}}(\omega)=\epsilon_{c}-\frac{\omega_{p}^{2}}{\omega(\omega+i \gamma)},
$$

to the observed plasma edges at low temperature in order (1) to determine the zero crossings in $\epsilon(\omega)$, which are the plasmon frequencies $\Omega_{p} \simeq \omega_{p} / \sqrt{\epsilon_{c}}$ and (2) then to get the unscreened plasma frequencies $\omega_{p}$ by estimating the core dielectric constant $\epsilon_{c}$. Standard RPA equations ${ }^{21}$ for the connection between $\omega_{p}$, crystallographic data, and the transfer integral subsequently yield the values given in Table I, where data for $\beta$ - $(\mathrm{ET})_{2} \mathrm{I}_{3}$ have been included to allow comparison.

All three materials have $t_{\|}: t_{\perp} \simeq 2.5: 1$, a rather modest anisotropy for organic conductors. This anisotropy corresponds within the simple model of Eq. (3) to a closed, cylindrical Fermi surface, but is not far from the crossover to an open Fermi surface.

Among the materials, the $\mathrm{AuI}_{2}{ }^{-}$compound appears to have the largest transfer integals as also evident from direct inspection of the plasma edges. With comparable anisotropies in $\beta$-(ET) ${ }_{2} \mathrm{AuI}_{2}$ and $\beta$-(ET) ${ }_{2} \mathrm{I}_{3}$ it follows that the Fermi level density-of-states $N(0)$ is bigger in the latter compound, which is consistent with a higher superconducting transition temperature,

$$
T_{c}=\left(\hbar \omega_{p h} / k_{B}\right) \exp [-1 / N(0) V],
$$

assuming otherwise unchanged parameters $\omega_{p h}$ and $V$.

The actual value of $N(0)$ will depend rather sensitively on the details of the band structure. In our crude model, $N(0) \simeq 2.5 \mathrm{eV}^{-1}$ (per molecule, both spin orientations) for $\beta$-(ET) ${ }_{2} \mathrm{I}_{3}\left[2.1 \mathrm{eV}^{-1}\right.$ for $\left.\beta-(\mathrm{ET})_{2} \mathrm{AuI}_{2}\right]$. In comparison, data for the $I_{3}$ - compound of paramagnetic susceptibili$\operatorname{ty}^{36} \chi_{s}$ and low-temperature electronic specific heat ${ }^{37} \mathrm{C}_{\mathrm{el}}$ would in a noninteracting electron-gas model lead to $N(0)=5.6$ and $5.0 \mathrm{eV}^{-1}$, respectively. The rather big 
TABLE I. Unscreened plasma frequencies and average transfer integrals for some $\beta$-(ET) ${ }_{2} X$ compounds at $T \simeq 30 \mathrm{~K}$. Data for $X=\mathrm{I}_{3}{ }^{-}$are from Ref. 21. $\epsilon_{c}=3.6-4.0$.

\begin{tabular}{ccccccc}
\hline \hline $\boldsymbol{X}$ & $\begin{array}{c}\omega_{p \|} \\
\mathrm{cm}^{-1}\end{array}$ & $\begin{array}{c}d_{\|} \\
\AA\end{array}$ & $\begin{array}{c}\omega_{p \perp} \\
\mathrm{cm}^{-1}\end{array}$ & $\begin{array}{c}d_{\perp} \\
\AA\end{array}$ & $\begin{array}{c}t_{\|} \\
\mathrm{eV}\end{array}$ & $\begin{array}{c}t_{\perp} \\
\mathrm{eV}\end{array}$ \\
\hline $\mathrm{I}_{3}{ }^{-}$ & 9600 & 4.62 & 5700 & 6.11 & 0.19 & 0.08 \\
$\mathrm{I}_{2} \mathrm{Br}^{-}$ & 9300 & 4.59 & 5300 & 6.11 & 0.18 & 0.07 \\
$\mathrm{AuI}_{2}{ }^{-}$ & 10300 & 4.55 & 5800 & 6.12 & 0.22 & 0.09 \\
\hline \hline
\end{tabular}

difference from the optical value suggests that $\chi_{s}$ is enhanced by Coulomb correlations (as frequently occur in organic conductors). $C_{\mathrm{el}}$ is presumably considerably enhanced by the electron-phonon interaction. ${ }^{37}$

In systems with considerable electron-electron interactions, the intraband absorption is generally reduced as compared to what is expected from the noninteracting electron gas. ${ }^{35}$ This decrease follows from a simple sum rule on the optical absorption, ${ }^{38}$ and can be used as a measure for the interaction strength. In Table II we compare the estimated integrated oscillator strength,

$$
I_{\sigma}=\frac{2}{\epsilon_{0} \pi} \int_{\text {intraband }} \sigma(\omega) d \omega,
$$

with $\omega_{p}^{2}$ from Table I. In the absence of interactions, and provided the absorption is located well below $\omega_{p}$, the two numbers should agree. Instead, we find in all cases a significant difference, which could be taken to indicate short-range interactions of the order of the bandwidth. ${ }^{35}$ This result is consistent with $\chi_{s}$ being enhanced $2-3$ times.

\section{B. Spectral features in the infrared}

We now discuss the distribution of oscillator strength in the infrared. Although the overall qualitative change with temperature is difficult to explain, some of the sharper features are readily understood as arising from the electron-molecular vibration coupling. For a nondegenerate orbital only the fully symmetric $A_{g}$ modes couple to the conduction electrons. ${ }^{39}$ The most important $A_{g}$ modes are listed in Table III (Ref. 40). The $A_{g}$ modes are not ordinarily infrared active. However, for a nonuniform molecular chain, they may by the symmetry lowering borrow intensity from the electrons resulting in spectral features with intensities related to the coupling strengths. The central $\mathrm{C}=\mathrm{C}$ stretching modes at $1300 \mathrm{~cm}^{-1}$ are

TABLE II. Integrated intraband oscillator strength for $\beta$ $(\mathrm{ET})_{2} X, T \simeq 30 \mathrm{~K}$, as compared with $\omega_{p}^{2}$. Data on $\beta-(\mathrm{ET})_{2} \mathrm{I}_{3}$ from Ref. 21 .

\begin{tabular}{clcr}
\hline$X$ & $\mathrm{I}_{3}{ }^{-}$ & $\mathrm{I}_{2} \mathrm{Br}^{-}$ & $\mathrm{AuI}_{2}{ }^{-}$ \\
\hline$\omega_{p \|}^{2}\left(10^{7} \mathrm{~cm}^{-2}\right)$ & 9.2 & 8.7 & 10.6 \\
$I_{\sigma \|}{ }^{\circ}\left(10^{7} \mathrm{~cm}^{-2}\right)$ & 6.2 & 5.7 & 7.9 \\
$\omega_{p \perp}^{2}\left(10^{7} \mathrm{~cm}^{-2}\right)$ & 3.3 & 2.8 & \\
$I_{\sigma \perp}\left(10^{7} \mathrm{~cm}^{-2}\right)$ & 2.0 & 1.8 & 3.4 \\
\hline
\end{tabular}

known to couple strongly in TTF-like molecules. ${ }^{41}$ Indeed, strong vibrational effects in this range are visible in all the stacking axis spectra (Figs. 4 and 7); the broken symmetry presumably arises from the dimerization. In the perpendicular direction the molecules have equivalent positions, hence the vibrational features are expected to be weak, consistent with the observed spectra (Figs. 5 and 8), although the strongly coupled $\mathrm{C}=\mathrm{C}$ modes are visible in the $\beta$-(ET) $)_{2} \mathrm{I}_{2} \mathrm{Br}$ spectra. A possible cause is the disorder introduced by the $\mathrm{I}_{2} \mathrm{Br}^{-}$ions. $^{25}$ [In $\beta$-(ET) ${ }_{2} \mathrm{AuI}_{2}$ the counterions are ordered.] Very similar observations may be made for the $430-460 \mathrm{~cm}^{-1}$ doublet, although in this case it is hardly visible in the $\mathbf{E} \perp$ (stacks) spectra for any of the compounds.

The low-temperature, far infrared conductivity spectra of highly conducting organic metals constitute a somewhat controversial subject. ${ }^{42}$ Different experimental groups do not always agree on their findings, but in a majority of cases the results point to the existence of narrow modes of possible collective origin centered at zero frequency $^{42}$ (i.e., the dc conductivity much exceeds what a reasonable extrapolation of the far infrared data would suggest.) In a few cases the low-frequency rise can be followed in a limited frequency range. ${ }^{42}$ In the present materials, although the far infrared is quite non-Drude, the extrapolated $\mathrm{dc}$ conductivities are in rather good agreement with measured values. Thus no narrow modes are implied at the temperatures investigated. The $35-\mathrm{GHz}$ stacking axis conductivity has for example been mea$\operatorname{sured}^{34}$ for $\beta-(\mathrm{ET})_{2} \mathrm{AuI}_{2}: \sigma_{\|}(T) \propto T^{2}$ and $\sigma_{\|}(30 \mathrm{~K}) \simeq 300$ $\Omega^{-1} \mathrm{~cm}^{-1}$ [compare Fig. 7(a)].

Thus the non-Drude low-temperature behavior is attributed to strong interaction effects, both electron-electron and electron-phonon (-vibration). That the stronger nonDrude character in the low-temperature $\beta$-(ET) ${ }_{2} \mathrm{AuI}_{2}$ spectra is a superconducting phenomenon seems rather unlikely, since it would imply strong fluctuations to $30 \mathrm{~K}$ in a material with $T_{c} \simeq 5 \mathrm{~K}$.

Finally, we note that the qualitative changes from 300 to $30 \mathrm{~K}$ in all spectra must clearly be connected to the low-frequency conductivity. At $300 \mathrm{~K}$ the carriers are localized on the dimers on the time scale of the spectroscopic measurement. Thus what is seen may basically be the intradimer transition with electron-phonon interference effects. The diminished thermal disorder at low temperature gradually allows the carriers to become delocalized, thus resulting in more Drude-like spectra. The detailed theoretical picture is unclear, but the data seem to indicate that electron-phonon as well as electron-electron interactions should be taken into account. 
TABLE III. Totally symmetric ( $\left.A_{g}\right)$ modes of BEDT-TTF, after Meneghetti et al. (Ref. 40).

\begin{tabular}{ccc}
\hline Mode no. & $\begin{array}{c}\text { Calculated frequency } \\
\left(\mathrm{cm}^{-1}\right)\end{array}$ & Character \\
\hline$v_{1}$ & 2965 & $\mathrm{C}-\mathrm{H}$ stretch \\
$v_{2}$ & 2926 & $\mathrm{C}-\mathrm{H}$ stretch \\
$v_{3}$ & 1600 & $\mathrm{C}=\mathrm{C}$ stretch \\
$v_{4}$ & 1549 & $\mathrm{C}=\mathrm{C}$ stretch \\
$v_{5}$ & 1445 & $\mathrm{H}-\mathrm{C}-\mathrm{H}$ bending \\
$v_{6}$ & 1289 & $\mathrm{C}-\mathrm{C}-\mathrm{H}$ bending \\
$v_{7}$ & 1195 & $\mathrm{C}-\mathrm{C}-\mathrm{H}$ bending \\
$v_{8}$ & 1028 & $\mathrm{C}-\mathrm{C}$ stretch, $-\mathrm{C}-\mathrm{H}$ bending \\
$v_{9}$ & 930 & $\mathrm{C}-\mathrm{C}$ stretch, C-C-H bending \\
$v_{10}$ & 880 & $\mathrm{C}-\mathrm{S}$ stretch \\
$v_{11}$ & 642 & $\mathrm{C}-\mathrm{S}$ stretch, C-C stretch \\
$v_{12}$ & 478 & $\mathrm{C}-\mathrm{S}$ stretch \\
$v_{13}$ & 457 & $\mathrm{C}-\mathrm{S}$ stretch \\
$v_{14}$ & 373 & Exterior ring deformation \\
$v_{15}$ & 294 & Exterior ring deformation \\
$v_{16}$ & 156 & Interior ring deformation \\
$v_{17}$ & 28 & Exterior ring deformation \\
\hline \hline
\end{tabular}

\section{CONCLUSIONS}

In this paper we have presented and discussed the infrared spectra of two $\beta$-(ET) ${ }_{2} X$ compounds. The data confirm the materials to be effectively two dimensional with a band-structure anisotropy of $\sim 2: 1$. Average transfer intergrals were derived for directions both along and perpendicular to the stacks. The anomalous roomtemperature spectra may be related to localization of the carriers on the dimerized stacks. At low temperature the spectra are closer to what is expected for basically metallic behavior, although important electron-phonon and electron-electron interactions lead to deviations from the simple Drude model. Estimates of the intraband oscillator strength further suggest that short range electronelectron Coulomb correlations cannot be neglected.

\section{ACKNOWLEDGMENTS}

The authors thank Daniel Christensen and Flemming Nicolaisen, H. C. Ørsted Institue, for the loan of the Bruker Fourier transform spectrometer and for advice on its use. Niels Thorup is acknowledged for determining the crystal morphology. Work at Argonne has been supported by the U.S. Department of Energy, Office of Basic Energy Sciences, Division of Material Science, Contract No. W-31-109-ENG-38, while D.B.T. acknowledges support from the Danish Natural Science Research Council and from the National Science Foundation, Solid State Chemistry, Grant No. DMR-8416511. C.S.J. would like to thank the Royal Danish Academy of Sciences and Letters for support.
*Permanent address: Department of Physics, University of Florida, Gainesville, Florida 32611.

${ }^{1} \mathrm{G}$. Saito, T. Enoki, K. Toriumi, and K. Inokuchi, Solid State Commun. 42, 557 (1982).

${ }^{2}$ S. S. P. Parkin, E. M. Engler, R. R. Schumaker, R. Lagier, V. Y. Lee, J. C. Scott, and R. L. Greene, Phys. Rev. Lett. 50, 270 (1983).

${ }^{3}$ E. B. Yagubskii, I. F. Shchegolev, V. N. Laukhin, P. A. Kononovich, M. V. Kartsovnik, A. V. Zvarykina, and L. I. Buravov, Pis'ma Zh. Eksp. Teor. Fiz. 39, 12 (1984) [JETP Lett. 39, 12 (1984)].

${ }^{4}$ K. Murata, M. Tokumoto, H. Anzai, H. Bando, G. Saito, K. Kajimura, and T. Ishiguro, J. Phys. Soc. Jpn. 54, 1236 (1985). See also, J. E. Schirber, L. J. Azevedo, J. F. Kwak, E. L. Venturini, M. A. Beno, H. H. Wang, and J. M. Williams, Solid State Commun. 59, 525 (1986).

${ }^{5}$ M. Tokumoto, K. Murata, H. Bando, H. Anzai, G. Saito, K. Kajimura, and T. Ishiguro, Solid State Commun. 54, 1031 (1985).

6V. B. Ginodman, A. V. Gudenko, I. I. Zasavitskii, and E. B.
Yagubskii, Pis'ma Zh. Eks. Teor. Fiz. 42, 384 (1985) [JETP Lett. 42, 472 (1985)].

${ }^{7}$ J. E. Schirber, L. J. Azevedo, J. F. Kwak, E. L. Venturini, P.C. W. Leung, M. A. Beno, H. H. Wang, and J. M. Williams, Phys. Rev. B 33, 1987 (1986).

${ }^{8}$ E. B. Yagubskii, I. F. Shchegolev, S. I. Pesotskii, V. N. Laukhin, P.A. Kononovich, M. V. Kartsovnik, and A. V. Zvarykina, Pis'ma Zh. Eksp. Teor. Fiz. 39, 275 (1984) [JETP Lett. 39, 328 (1984)].

${ }^{9}$ J. M. Williams, H. W. Wang, M.A. Beno, T. J. Emge, L. M. Sowa, P. T. Copps, F. Behroozi, L. N. Hall, K. D. Carlson, and G. W. Crabtree, Inorg. Chem. 23, 3839 (1984).

${ }^{10}$ H. H. Wang, M. A. Beno, U. Geiser, M. A. Firestone, K. S. Webb, L. Nuñez, G. W. Crabtree, K. D. Carlson, J. M. Williams, L. J. Azevedo, J. F. Kwak, and J. E. Schirber, Inorg. Chem. 24, 2465 (1985).

${ }^{11}$ V. F. Kaminskii, T. G. Prokhorova, R. P. Shibaeva, and E. B. Yagubskii, Pis'ma Zh. Eksp. Teor. Fiz. 39, 15 (1984) [JETP Lett. 39, 17 (1984)]

12J. M. Williams, T. J. Emge, H. H. Wang, M. A. Beno, P. T. 
Copps, L. N. Hall, K. D. Carlson, and G. W. Crabtree, Inorg. Chem. 23, 2558 (1984).

${ }^{13}$ K. Mortensen, C. S. Jacobsen, K. Bechgaard, K. Carneiro, and J. M. Williams, Mol. Cryst. Liq. Cryst. 119, 401 (1985).

${ }^{14}$ T. Mori, A. Kobayashi, Y. Sasai, H. Kobayashi, G. Saito, and H. Inokuchi, Chem. Lett. 1984, 957 (1984).

${ }^{15}$ M.-H. Whango, J. M. Williams, P. C. W. Leung, M. A. Beno, T. J. Emge, H. H. Wang, K. D. Carlson, and G. W. Crabtree, J. Am. Chem. Soc. 107, 5815 (1985).

${ }^{16}$ B. Koch, H. P. Geserich, W. Ruppel, D. Schweitzer, K. H. Dietz, and H. J. Keller, Mol. Cryst. Liq. Cryst. 119, 343 (1985).

${ }^{17}$ H. Tajima, K. Yakushi, H. Kuroda, and G. Saito, Solid State Commun. 56, 159 (1985).

${ }^{18}$ H. Kuroda, K. Yakushi, H. Tajima, and G. Saito, Mol. Cryst. Liq. Cryst. 125, 135 (1985).

${ }^{19}$ M. G. Kaplunov, E. B. Yagubskii, L. P. Rosenberg, and Yu. G. Borodko, Phys. Status Solidi A 89, 509 (1985).

${ }^{20}$ T. Sugano, K. Yamada, G. Saito, and M. Kinoshita, Solid State Commun. 55, 137 (1985).

${ }^{21}$ C. S. Jacobsen, J. M. Williams, and H. H. Wang, Solid State Commun. 54, 937 (1985). Note that the polarizations \| and $\perp$ in that paper should be interchanged [Erratum: Solid State Commun. 57, No. 8, p. i (1986)].

${ }^{22}$ H. Tajima, H. Kanbara, K. Yakushi, H. Kuroda, and G. Saito, Solid State Commun. 57, 911 (1986).

${ }^{23}$ H. Tajima, H. Kanbara, K. Yakushi, H. Kuroda, and G. Saito, Proceedings of the International Conference on Synthetic Metals, Kyoto, 1986 [Synth. Met. 19, 137 (1987)].

${ }^{24}$ C. S. Jacobsen, D. B. Tanner, J. M. Williams, and H. H. Wang, Proceedings of the International Conference on Synthetic Metals, Kyoto, 1986 [Synth. Met. 19, 125 (1987)].

${ }^{25}$ T. J. Emge, H. H. Wang, M. A. Beno, P. C. W. Leung, M. A. Firestone, H. C. Jenkins, J. D. Cook, K. D. Carlson, J. M. Williams, E. L. Venturini, L. J. Azevedo, and J. E. Schirber, Inorg. Chem. 24, 1736 (1985).

${ }^{26}$ C. S. Jacobsen, D. B. Tanner, and K. Bechgaard, Phys. Rev. B 28, 7019 (1983). [TMTSF is an abbreviation of tetramethyltetraselenafulvalene $\left(\mathrm{C}_{10} \mathrm{H}_{12} \mathrm{Se}_{4}\right)$ with the structural formula 2,2'-bi(4,5-dimethyl-1,3-diselenole-2-ylidene); TMTTF is tetramethyltetrathiafulvalene $\left(\mathrm{C}_{10} \mathrm{H}_{12} \mathrm{~S}_{4}\right)$ of the same structure.]

${ }^{27}$ See, for example, F. Wooten, Optical Properties of Solids (Academic, New York, 1972).

28 J. B. Torrance, B. A. Scott, and F. B. Kaufman, Solid State Commun. 17, 1369 (1975)

${ }^{29}$ P. F. Maldague, Phys. Rev. B 16, 2437 (1977).

30J. Tanaka, M. Tanaka, C. Tanaka, T. Ohno, T. Takabe, and H. Anzai, Ann. N.Y. Acad. Sci. 313, 256 (1978). [TCNQ is an abbreviation for 7,7,8,8-tetracyanoquinodimethane $\left(\mathrm{C}_{12} \mathrm{H}_{4} \mathrm{~N}_{4}\right)$ with the structural formula 2,2'-(2,5cyclohexadiene-1,4-diylidene)bis(propanedinitrile-2-ylidene).]

${ }^{31}$ P. A. Lee, T. M. Rice, and P. W. Anderson, Solid State Commun. 14, 703 (1974).

32J. Hubbard, Phys. Rev. B 17, 494 (1978).

${ }^{33}$ H. Hinkelmann and H. G. Reik, Solid State Commun. 16, 567 (1975).

${ }^{34}$ D. B. Tanner, C. S. Jacobsen, J. M. Williams, and H. H. Wang (unpublished).

${ }^{35}$ C. S. Jacobsen, J. Phys. C 19, 5643 (1986).

${ }^{36}$ V. A. Merzhanov, E. E. Kostyuchenko, O. E. Faber, I. F. Shchegolev, and E. B. Yagubskii, Zh. Eksp. Teor. Fiz. 89, 292 (1985) [Sov. Phys._JETP 62, 165 (1985)].

${ }^{37}$ G. R. Stewart, J. O'Rourke, G. W. Crabtree, K. D. Carlson, H. H. Wang, J. M. Williams, F. Gross, and K. Andres, Phys. Rev. B 33, 2046 (1986).

${ }^{38}$ S. Mazumdar and Z. G. Soos, Phys. Rev. B 23, 2810 (1981).

${ }^{39}$ C. B. Duke, N. O. Lipari, and L. Pietronero, Chem. Phys. Lett. 30, 415 (1975).

${ }^{40}$ M. Meneghetti, R. Bozio, and C. Pecile, J. Phys. (Paris) 47, 1377 (1986).

${ }^{41}$ See, for example, R. Bozio and C. Pecile in The Physics and Chemistry of Low-Dimensional Solids, edited by L. Alcàcer, (Reidel, Dordrecht, 1980), p. 169.

${ }^{42} \mathrm{~T}$. Timusk, in Low-Dimensional Conductors and Superconductors, edited by L. Caron and D. Jérome (Plenum, New York, 1987), and references therein. 